\title{
Análisis clínico y citogenético de un caso de trastorno del desarrollo sexual testicular XX con SRY negativo
}

\author{
Perla Cristina Salgado ${ }^{\text {- Adriana del Pilar Ramírez }}{ }^{\mathbf{b}}$ - Diana Marcela Gallo \\ Orjuelac - Carlos Alberto Garzón ${ }^{d}$ - Yaqueline Ladino - Orietta Ivonne Beltrán ${ }^{f}$
}

Resumen: El trastorno del desarrollo sexual (TDS) testicular XX es una patología que se presenta en un individuo con cariotipo 46,XX con un fenotipo anatómico de genitales externos masculinos, que pueden variar desde la normalidad hasta la ambigüedad genital. Clínicamente se han descrito dos subgrupos de hombres 46,XX con SRY-negativos y SRY-positivos, dependiendo de la presencia o no del gen SRY que normalmente se encuentra en el cromosoma Y participando en la determinación testicular. En este artículo se describen los antecedentes personales y los hallazgos clínicos de un infante con anomalías del meato urinario en el cual se identificó un complemento cromosómico 46,XX. También, se realizó hibridación in situ fluorescente en linfocitos de sangre periférica que demostró la ausencia del gen SRY y confirmó la presencia de dos cromosomas $X$.

Palabras clave: trastorno del desarrollo sexual testicular 46,XX; varón 46,XX; SRY- negativo; hibridación in situ fluorescente

Fecha de recepción: 23 de febrero de 2018 Fecha de aprobación: 1 de noviembre de 2018

Cómo citar: Salgado PC, Ramírez A del P, Gallo Orjuela DM, Garzón CA, Ladino Y, Beltrán OI. Análisis clínico y citogenético de un caso de trastorno del desarrollo sexual testicular XX con SRY negativo. Revista Med. 27(1):45-52. Disponible en: https://revistas.unimilitar.edu.co/index.php/rmed/article/view/4753

a Médico cirujano, Fuerza Aérea Colombia. Bogotá, Colombia.

b Bacterióloga, magíster en Genética Humana, Grupo de investigación BioGenEtica \& BioDerecho, Facultad de Medicina, Universidad Militar Nueva Granada. Bogotá, Colombia

c Cirujana Maxilofacial, Odontóloga y Médica, Departamento de Cirugía Maxilofacial, Clínica Partenón, Bogotá, Colombia

d Médico, residente de Pediatría, Facultad de Medicina Universidad Militar Nueva Granada, Bogotá, Colombia.

e Médica, Magister en Genética Humana, Grupo de Investigación GenHOMI, Fundación HOMI - Hospital Pediátrico de la Misericordia, Bogotá, Colombia. Maestría en Genética Humana, Universidad Nacional de Colombia, Bogotá, Colombia.

f Médica, Magister en Genética Humana, Grupo de Investigación en BioGenEtica \& BioDerecho, Facultad de Medicina Universidad Militar Nueva Granada, Bogotá, Colombia. Grupo de Investigación GenHOMI, Fundación HOMI - Hospital Pediátrico de la Misericordia, Bogotá, Colombia. 


\section{Clinical and Cytogenetic Analysis of a Case of SRY-Negative XX Testicular Disorder of Sex Development}

Resumo: XX testicular disorder of sex development (DSD) is a pathology that occurs in an individual with a 46,XX karyotype and an anatomical phenotype of male external genitalia, which may vary from normal to ambiguous. Clinically, two subgroups of SRY-negative and SRY-positive, 46, XX men have been described, depending on the presence of the SRY gene that is normally found on the Y chromosome participating in testicular determination. This article describes the personal history and clinical findings of an infant with urethral meatus abnormalities in whom a 46,XX chromosome set was identified. Also, fluorescent in situ hybridization was performed in peripheral blood lymphocytes which demonstrated the absence of the SRY gene and confirmed the presence of two X chromosomes.

Keywords: 46,XX testicular disorder of sex development; 46,XX male; SRY-negative; fluorescent in situ hybridization

\section{Análise clínica e citogenética de um caso de transtorno do desenvolvimento sexual testicular XX com SRY-negativo}

Resumo: O transtorno do desenvolvimento sexual (TDS) testicular XX é uma patologia apresentada em um indivíduo com cariótipo 46,XX com um fenótipo anatômico de genitais externos masculinos, que podem variar da normalidade à ambiguidade genital. Clinicamente, são descritos dois subgrupos de homens 46,XX com SRY-negativos e SRY-positivos, dependendo da presença ou não do gene SRY que normalmente se encontra em Y cromossomo participando da determinação testicular. Neste artigo, são descritos os antecedentes pessoais e os achados clínicos de uma criança com anomalias de meato urinário em que foi identificado um complemento cromossômico 46,XX. Além disso, foi realizada hibridação in situ fluorescente em linfócitos de sangue periférico que demonstrou a ausência do gene SRY e confirmou a presença de dois cromossomos $X$.

Palavras-chave: transtorno do desenvolvimento sexual testicular 46,XX; homem 46,XX; SRY-negativo; hibridação in situ fluorescente 


\section{Introducción}

Los trastornos del desarrollo sexual (TDS) comprenden un grupo de afecciones congénitas caracterizadas por un componente cromosómico, gonadal y anatómico atípico (1). Se estima que entre el 1 y el $2 \%$ de los nacidos vivos puede padecer este tipo de anomalía congénita (1-3). Dentro de la clasificación de los TDs (1) se ha descrito una patología denominada TDs testicular 46,XX, la cual consta de la evidencia de un cariotipo con complemento cromosómico 46,XX en un individuo con genitales externos masculinos, que van desde una presentación clínica normal hasta genitales ambiguos, presencia de dos testículos, ausencia de estructuras derivadas del conducto de Müller e infertilidad debida a azoospermia (2-5).

La diferenciación del sexo durante la etapa embrionaria se produce gracias al desarrollo de las crestas gonadales indiferenciadas para formar los ovarios o los testículos. El gen SRY (de las siglas en inglés: sex-determining region $Y$ ) ha demostrado ser crucial en la diferenciación testicular y normalmente se localiza en la región distal del brazo corto del cromosoma Y (Yp11.2) (3, 4); sin embargo, el gen SRY pueden encontrarse translocado en uno de los cromosomas $\mathrm{X}$ en un porcentaje aproximado del $80 \%$ de los pacientes masculinos con complemento cromosómico 46,XX (5).

La prevalencia del TDs testicular 46,XX es de 1 en $20.000(1,5)$. En el $80 \%$ de los casos con TDS testicular 46,XX los individuos son positivos para el gen SRY, identificado usualmente a través de hibridación in situ fluorescente (FISH) o hibridación genómica comparativa con microarreglos (CGH) (4, 5). En este artículo se describe el caso clínico de un infante con TDS testicular 46,XX con SRY negativo.

\section{Metodología}

\section{Revisión de caso clínico}

Se describe un reporte de caso sobre el seguimiento clínico de un niño con hipospadia que cursa con un TDs testicular 46,XX.

\section{Hibridación in situ fluorescente}

\section{Muestras}

Se obtuvieron muestras de sangre periférica de tres individuos: la muestra del paciente evaluado en la consulta externa de genética clínica pediátrica (caso). Además, se utilizaron dos muestras adicionales como controles experimentales de donantes voluntarios sanos: una muestra de un individuo de sexo masculino con descendencia (control positivo) y una muestra de un individuo femenino con descendencia (control negativo).

\section{Procedimiento}

Previo consentimiento informado se realizó cultivo de linfocitos de sangre periférica para obtener a las 72 horas, extendidos cromosómicos en lámina portaobjetos; posteriormente, se hizo protocolo de hibridación Vysis SRY/CEPX FISH Probe Kit siguiendo las recomendaciones del fabricante (6). Luego, se inició procesamiento de la muestra con desnaturalización del DNA a $73^{\circ} \mathrm{C}$ durante cinco minutos, seguido de solución de etanol al $70 \%$, $80 \%$ y $100 \%$ durante un minuto en cada una de ellas. Se adicionó sonda marcada para el gen del SRY (LSI) y la sonda centromérica del cromosoma $\mathrm{X}$ (CEPX, DXZ1), y se dejó hibridar a $37^{\circ} \mathrm{C}$ por doce horas. Se realizaron lavados con solución poshibridación a $73^{\circ} \mathrm{C}$ por dos minutos, seguidos de un segundo lavado con solución poshibridación. Se adicionó colorante DAPI y se visualizaron los núcleos de los linfocitos a través de un microscopio de fluorescencia marca Nikon Eclipse E-80i con filtros de Spectrum Green para la sonda CEPX (DXZ1, Xp11.1-q11.1, Vysis) y Spectrum Orange para la sonda LSI SRY (Yp11.3). Al final, se hizo el montaje con controles positivos y negativos para las sondas utilizadas $(3,6,7)$.

\section{Resultados}

\section{Descripción de caso}

Asiste a la consulta de genética un varón de 28 días de nacido por cuadro de hipospadia y pene pequeño, fruto de unión no consanguínea de padres sanos, primera gestación, controlada sin exposición a teratógenos, sin antecedente de endocrinopatías, 
ni exposiciones hormonales durante la gestación. No hay historia familiar de síndromes genéticos, genitales ambiguos o muerte neonatal temprana. Durante el control prenatal a los siete meses de gestación se diagnosticó por ecografía hipospadias. El varón nació por cesárea de urgencia a las 36 semanas, por disminución de los movimientos fetales, con un peso de 3170 gr y talla al nacer de $51 \mathrm{~cm}$. Presentó aspiración de líquido amniótico, por lo que necesitó hospitalización en unidad neonatal por ocho días. En evaluación clínica a los 28 días de vida el paciente tenía peso y talla normales (percentil 25-50), se mostró activo, reactivo, normocéfalo, con simetría facial, pero evidenciando genitales masculinos con presencia de epispadias (meato funcional) e hipospadias con meato proximal no funcional, con testículos en bolsa escrotal. El resto del examen físico no presentó alteraciones.

Luego, se realizó cariotipo convencional en bandeo G con resultado 46,XX confirmado en dos muestras independientes. estos estudios se complementaron con una ecografía testicular la cual evidencia ambos testículos con contornos bien definidos y ecogenicidad homogénea. Adicionalmente, se valoró el tracto urinario por ecografía renal y de vías urinarias, lo cual mostró riñones, uréteres y vejiga normales.

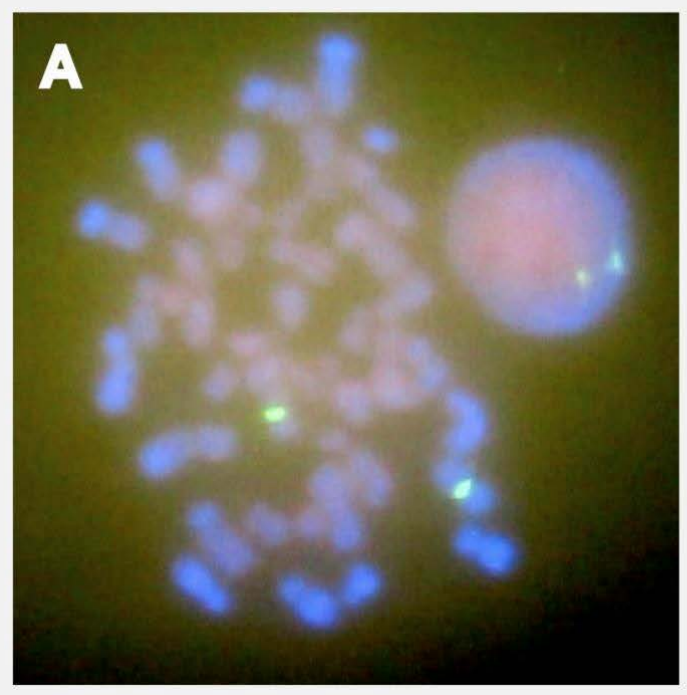

\section{Hibridación in situ fluorescente}

Se realizaron las observaciones con el microscopio de fluorescencia Nikon eclipse $80 \mathrm{i}$, y para la captura de las señales fluorescentes se utilizó el programa de Lucia kario FISH. Se observaron dos fluorescencias verdes (Spectrum Green) de la sonda que contiene la secuencia del satélite alfa de la región Dxzl que es específica para el cromosoma $\mathrm{X}$ (ver figura 1). No se observó fluorescencia naranja (Spectrum Orange) de la región cromosómica del gen SRY en 100 metafases y núcleos analizados. Su constitución cromosómica fue: 46,XX. ISH CEPX (DXZ1, Xp11.1-q11.1x2), (sRY-)(Yp11.3-). Los resultados de los controles positivos fueron concordantes con el fenotipo y sexo de los donantes. En los resultados de la muestra del control positivo de individuo masculino, se observó una fluorescencia verde (Spectrum Green) de la sonda que contiene la secuencia del satélite alfa de la región Dxz1 y se observó una fluorescencia naranja (Spectrum Orange) de la región cromosómica del gen SRY (figura 1). En los resultados de la muestra del control negativo de individuo femenino, se pudieron ver las dos fluorescencias verdes (Spectrum Green) de la sonda que contiene la secuencia del satélite alfa de la región Dxz1 del cromosoma X.

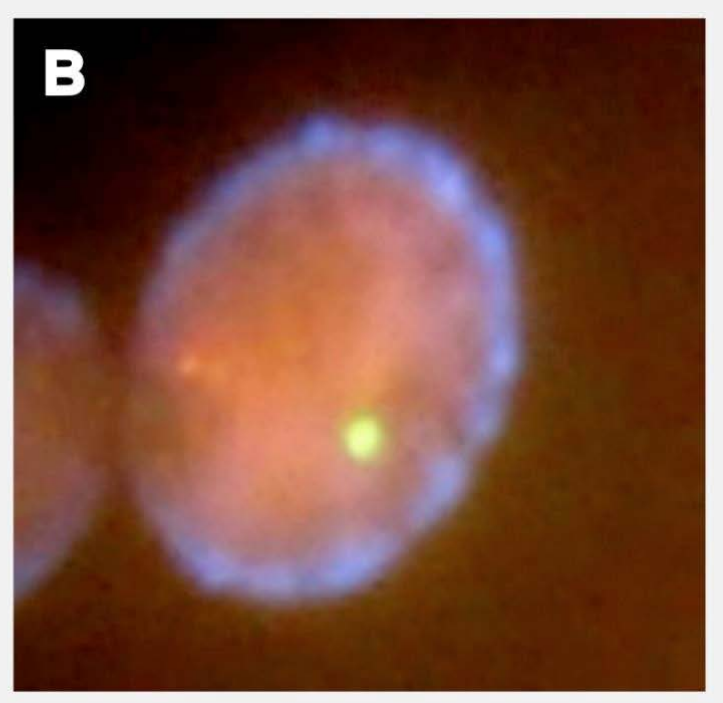

Figura 1. Hibridación in situ fluorescente para identificación del gen SRY: a. muestra del paciente, se observan dos fluorescencias verdes (Spectrum Green) en el núcleo en interfase y en los cromosomas metafásicos, b. control positivo de individuo masculino sano, se observan fluorescencia verde (Spectrum Green) y fluorescencia naranja (Spectrum Orange) en el núcleo en interfase. 


\section{Discusión}

El desarrollo sexual es uno de los procesos biológicos más importantes y transcendentales, que conlleva características específicas morfológicas, fisiológicas y comportamentales indispensables para la reproducción $(3,4)$. Inicialmente, se consideró que la presencia de genes del cromosoma $Y$ en los varones era la causa del proceso de formación hacia testículo de la gónada indiferenciada (8-10). Esta observación condujo a la identificación del gen SRY en el locus Yp11.2, el cual codifica una proteína que actúa como factor de transcripción de la familia de proteínas de unión al DNA con dominio conservado de alta movilidad HMG (siglas en inglés de High Mobility Group) $(8,11)$. Su proteína es el factor determinante testicular (TDF), el cual gracias al dominio HMG puede unirse a sitios específicos de la curvatura menor del DNA, lo que induce un cambio estructural de la cromatina que facilita el ensamblaje de complejos multiproteícos, y esto regula la expresión génica por activación o represión de diversos genes blancos (10-12). Sin embargo, se han descrito diferentes genes y vías de señalización que participan en la determinación de la gónada bipotencial hacia testículo y ovario, y en la actualidad se ha reconocido que en los mamíferos el desarrollo sexual es un proceso complejo, dinámico y controlado genéticamente $(1,5,13,14)$.

Gracias a los estudios de genómica estructural y funcional de los TDs en humanos y el entendimiento de mecanismos moleculares, el enfoque clínico y el conocimiento de las bases moleculares de los TDS han sufrido grandes cambios en la última década. Así, pues, en 2006 se modificó la clasificación de los TDs a través de un consenso entre expertos en el tema $(1,2)$. Dada la complejidad de desarrollo sexual y las percepciones del sexo, el género y la sexualidad es de esperarse que los individuos afectados con TDS requieran un manejo interdisciplinario que incluya la participación de endocrinología, urología, cirugía, genética, psicología, entre otras profesiones de la salud $(1,4)$.

Como se mencionó, el desarrollo de las gónadas (ovarios y testículos) es regulado por una cascada molecular, hacia la diferenciación masculina o femenina específica, incluyendo la presencia del gen SRY que actúa como "interruptor genético" y es determinante en la interacción de genes necesarios en la determinación del sexo masculino (11, 12). El caso de esta investigación corresponde a un individuo con TDS testicular $\mathrm{xx}$, con dos cromosomas sexuales $\mathrm{xx}$ identificados inicialmente por citogenética convencional en bandeo G, cuyo complemento cromosómico normalmente se encuentra en fenotipo femenino, pero en esta entidad se presenta un fenotipo masculino. Con este hallazgo se consideró que existía una falta de coincidencia entre el sexo cromosómico y el sexo fenotípico. Posteriormente, los resultados del FisH mostraron la ausencia total de fluorescencia de la sonda que hibrida en el gen SRY, lo que permitió clasificar al paciente dentro del $20 \%$ de los casos con TDs testicular XX con gen SRY negativo.

Hay dos subgrupos de hombres 46,xx: SRY-negativos (rango entre 10 y $20 \%$ ) y sRY-positivos (rango entre 80 y $90 \%$ ), esto dependiendo de la presencia o no del gen SRY, que normalmente se encuentra en cromosoma $\mathrm{Y}$ e interviene en la diferenciación testicular $(5,7)$. Los pacientes que presentan positividad para el gen SRY por lo general evidencian una translocación en el cromosoma $\mathrm{X}$ o cualquier otro autosoma $(5,15,16,17)$. Esto fue descartado por la ausencia de SRY documentada en la hibridación in situ fluorescente locus específica en sangre periférica que se le hizo al paciente (ver figura 1).

El mecanismo por el cual en los pacientes con SRY-negativo se presenta la inducción testicular tiene algunas hipótesis: 1) mosaicismo escondido para el gen SRY; 2) mutaciones autosómicas o ligadas al cromosoma $\mathrm{X}$ en genes que reprimen la vía masculina, y 3) expresión alterada de otros genes que son determinantes en la cascada de la diferenciación sexual, como RSPO1, DAX1, SOX9, DAX1, SF1, WT1, GATA4, WNT4, FGF9, SOX3, SOX10, ROCK1 y DMRT, entre otros $(5,7,13,18,19,20)$.

En este caso, y como lo indica la literatura, individuos 46,XX SRY-negativos en raras ocasiones tienen masculinización completa y en muchos casos presentan diferencias fenotípicas, como es el caso del paciente en estudio con una presentación poco común de hipospadia y epispadia concomitantes, y para el cual se recomienda ampliar la búsqueda de la etiología enfocándose en genes adicionales que participan 
en el desarrollo testicular $(5,21,22)$. Los individuos con este tipo de afecciones por lo general son criados como varones y tienen identidad de género masculino; aproximadamente el $85 \%$ de los individuos después de la pubertad presenta vello púbico normal, pene de tamaño normal, testículos pequeños, ginecomastia y esterilidad, resultante de la azoospermia (21-23). Usualmente, los individuos requieren en la adolescencia seguimiento clínico por la probabilidad de presentar hipogonadismo hipergonadotrófico (5, 17, 21, 23).

La evidencia clínica identificada en casos de pacientes con TDS testicular XX con SRY ausente (23-26) y en modelos in vivo en ratones (27) sustenta el hecho de que la sobreexpresión de genes de la familia sox, relacionados con el dominio HMG del SRY, expliquen una parte de los mecanismos moleculares de este subgrupo clínico (24-27). Asimismo, mutaciones con pérdida de función en los genes de la diferenciación del ovario, principalmente por genes de la vía de señalización de Wnt, pueden explicar este trastorno $(5,13,23)$. Debido a la heterogeneidad alélica descrita, el mecanismo de herencia para los individuos 46,XX con SRY negativo es desconocido y dependiente del tipo de mutación causal que pudiera identificarse $(5,13)$. La relación del fenotipo-genotipo del paciente podría tener otras explicaciones como mosaicismo no detectado, ya que las muestras por estudiar son diferentes al tejido testicular; también puede ser una pérdida del cromosoma $\mathrm{Y}$ en etapas tempranas de la embriogénesis (28).

El uso de la hibridación in situ flourescente en el diagnóstico molecular de TDs ha demostrado ser una técnica eficiente para la localización rápida de los genes como el SRY, que tiene una sola copia en el DNA. Otra de las ventajas de su uso es que permite una conservación adecuada de los extendidos y la visualización de las señales en las metafases y en los núcleos donde se pueden revisar célula a célula (29). Adicionalmente, es una técnica con alta especificidad, de fácil estandarización, costo relativamente bajo, en la cual el valor predictivo positivo es alto cuando se cuenta con una preparación adecuada de las muestras, equipos correctos y la experticia del analista de citogenética. En nuestro estudio se observó una concordancia con el resultado de cariotipo convencional, con respecto a la composición cromosómica $46, \mathrm{xx}$, con la ventaja de poder demostrar la ausencia del gen SRY (6, 30,31 ).

Sin embargo, la incertidumbre generada por la no identificación de la etiología molecular de los TDS en general, y en nuestro caso particular, plantea incomodidades a los pacientes y sus familiares, dado que no es posible ofrecer un asesoramiento genético familiar completo y adecuado (5, 31-34). Así, pues, se considera indispensable el seguimiento clínico de estos pacientes, ya que, si bien es cierto que los hombres $46, \mathrm{xx}$ presentan expresividad variable, todos los pacientes son infértiles por la ausencia de las regiones de la azoospermia AZFa, AZFb y AZFc, ubicada en el brazo largo del cromosoma Y (5). No obstante, en el futuro cercano, con el uso de nuevas tecnologías diagnósticas como la CGH, paneles de secuenciamiento de nueva generación para evaluar simultáneamente varios genes e incluso el análisis de exoma completo están facilitando el aumento del porcentaje de diagnósticos confirmados de manera temprana, para definir el manejo integral de los pacientes con TDs (31-35).

\section{Conflicto de interés}

Los autores no declaramos conflicto de interés.

\section{Financiación}

Los recursos para la financiación de este artículo provienen de un proyecto de iniciación científica aprobado y financiado por la Vicerrectoría de Investigaciones de la Universidad Militar Nueva Granada con código PICMED2011 y el proyecto INVMED1828, vigencia 2015. Este artículo es producto derivado del proyecto INVMED1828 financiado por la Vicerrectoría de Investigaciones de la Universidad Militar Nueva Granada, vigencia 2015.

\section{Agradecimientos}

A los padres del paciente, a los especialistas participantes de la Junta de Desórdenes del Desarrollo Sexual de la Fundación номі, Hospital Pediátrico la Misericordia, especialmente a la doctora Clara Arteaga, médica genetista; al doctor Camilo 
Orjuela, urólogo pediatra, y al doctor Mauricio Coll, endocrinólogo pediatra, por compartir su conocimiento y motivar nuestro aprendizaje por este grupo de patologías. También agradecemos el apoyo logístico de Carlos Daza, biólogo y coordinador del Laboratorios de la Facultad de Medicina de la Universidad Militar Nueva Granada, y de María del Pilar Pérez, química laboratorista del Laboratorio de Genética de la Facultad de Medicina de la Universidad Militar Nueva Granada. Por último, agradecemos a los donantes voluntarios sanos.

\section{Referencias}

[1] Lee P, Houk C, Ahmed S, Hughes I. Consensus statement on management of intersex disorders. International Consensus Conference on Intersex. Pediatrics. 2006 agos.;118(2):e488-500.

[2] Pasterski V, Prentice P, Hughes I. Impact of the consensus statement and the new DSD classification system. Best Pract. Res Clin Endocrinol Metab. 2010 abr.;24(2):187-95.

[3] McClelland K, Bowles J, Koopman P. Male sex determination: Insights into molecular mechanisms. Asian J Androl. 2012 en.;14(1):164-171.

[4] Del Castillo V, Uranga R, Zafra G. Genética clínica. 1. ${ }^{a}$ ed. Ciudad de México: Manual Moderno; 2012. Cap. 9, Aspectos genéticos, moleculares y celulares de la diferenciación gonadal; p. 269-280. Cap. 10, Desórdenes del desarrollo sexual; p. 281-293.

[5] Délot E, Vilain E. Nonsyndromic 46,XX Testicular Disorders of Sex Development. [Internet]. Seatlle: University of Washington; 2003-2015. Disponible en: http://www.ncbi.nlm.nih.gov/books/NBK1416/

[6] Abbott Molecular. Vysis SRY Probe LSI SRY Spectrum Orange/CEP X Spectrum Green. Disponible en: https://www.molecular.abbott/us/en/chromosome/Y

[7] Li T-F, Wu Q-Y, Zhang C, Li W-W, Zhou Q, Jiang W-J, et al. 46,XX testicular disorder of sexual development with SRY-negative caused by some unidentified mechanisms: a case report and review of the literature. BMC Urol. 2014:14:104.

[8] Sinclair A, Berta P, Palmer M, Hawkins J, Griffiths B, Smith M, et al. A gene from the human sex-determining region encodes a protein with homology to a conserved DNA-binding motif. Nature. 1990 jul.;346(6281):240-4.
[9] Mittwoch U. Sex determination and sex reversal: genotype, phenotype, dogma and semantics. Hum Genet. 1992 jul.;89(5):467-479.

[10] Goodfellow P, Lovell R. SRY and sex determination in mammals. Annu Rev Genet. 1993;27:71-92.

[11] GeneCards. SRY Gene [Internet]. Disponible en: https://www.genecards.org/cgi-bin/carddisp.pl?gene=SRY\&keywords $=$ sry

[12] SRY-Sex-determining region $Y$ protein-Homo sapiens (Human)-SRY gene \& protein [Internet]. Disponible en: http://www.uniprot.org/uniprot/ Q05066\#entry_information

[13] Ono M, Harley V. Disorders of sex development: new genes, new concepts. Nat Rev Endocrinol. 2013 febr.;9(2):79-91.

[14] Bashamboo A, McElravey K. Human sex-determination and disorders of sex- development (DSD). Semin Cell Dev Biol. 2015 sept.;45:77-83.

[15] Evans HJ, Buckton KE, Spowart G, Carothers AD. Heteromorphic X chromosomes in 46, XX males: evidence for the involvement of X-Y interchange. Hum Genet. 1979 may.;49(1):11-31.

[16] Fechner P, Marcantonio S, Jaswaney V, Stetten G, Goodfellow P, Migeon C. The role of the sex-determining region $\mathrm{Y}$ gene in the etiology of $46, \mathrm{XX}$ maleness. J Clin Endocrinol Metab. 1993;76(3):690-5.

[17] Ergun-Longmire B, Vinci G, Alonso L, Matthew S, Tansil S, Lin-Ka K. Clinical, hormonal and cytogenetic evaluation of 46,XX malies and review of the literature. J Pediatr Endocrinol Metab. 2005 ag.;18(8):739-48.

[18] Huang B, Wang S, Ning Y, Lamb A, Bartley J. Autosomal XX sex reversal caused by duplication of SOX9. Am J Med Genet. 1999 dic. 3;87(4):349-53.

[19] Cox J, Willatt L, Homfray T, Woods G. A SOX9 duplication and familial 46,XX developmental testicular disorder. N Engl J Med. 2011 en. 6;364:91-3.

[20] Temel S, Gulten T, Yakut T, Saglam H, Kilic N, Bausch $\mathrm{E}$, et al.. Extended pedigree with multiple cases of XX sex reversal in the absence of SRY and of a mutation at the SOX9 locus. Sex Dev. 2007;1(1):24-34.

[21] Grigorescu-Sido A, Heinrich U, Grigorescu P, Jauch A, Hager H, Vogt $\mathrm{P}$, et al. Three new 46,XX male patients: a clinical, cytogenetic and molecular analysis. J Pediatr Endocrinol Metab. 2005 febr.;18(2):197-203.

[22] Chiang H, Wu Y, Wu C, Hwang J. Cytogenic and molecular analyses of 46,XX male syndrome with clinical comparison to other groups with testicular azoospermia of genetic origin. J Formos Med Assoc. 2013 febr.;112(2):72-8. 
[23] Arboleda VA, Sandberg DE, Vilain E. DSDs: genetics, underlying pathologies and psychosexual differentiation. Nat Rev Endocrinol. 2014 oct.;10(10):603-615.

[24] Sutton E, Hughes J, White S, Sekido R, Tan J, Arboleda $\mathrm{V}$, et al.. Identification pf SOX 3 as an XX male sex reversal gene in mice and humans. J Clin Invest. 2011 en.;121(1):328- 341.

[25] Kojima Y, Hayashi Y, Mizuno K, Sasaki S, Fukui Y, Koopman P, et al. Up-regulation of SOX9 in human sex-determining región on the $\mathrm{Y}$ chromosome (SRY)-negative XX males. Clin Endocrinol (Oxf). 2008 may.:68(5):791-9.

[26] Refai O, Friedman A, Terry L, Jewett T, Pearlman A, Perle MA, et al. De novo 12;17 translocation upstream of SOX9 resulting in 46,XX testicular disorder of sex development. Am J Med Genet A. 2010 febr.;152A(2):422-6.

[27] Polanco JC, Wilhelm D, Davidson TL, Knight D, Koopman P. Sox10 gain-of-function causes XX sex reversal in mice: implications for human 22q-linked disorders of sex development. Hum Mol Genet. 2010 febr. 1;19(3):506-516.

[28] Marquet F, Verloes A, Beckers A. [Clinical case of the month. A male with 46,XX karyotype]. Rev Med Liege. 1998 sept.;53(9):515-517.
[29] Gao JL, Nie Y, Ding XP. Primed in situ labeling for detecting single-copy genes. Genet. Mol. Res. 2011 ag. 26;10(3):1884-90.

[30] Garimberti E, Tosi S. Fluorescence in situ hybridization (FISH), basic principles and methodology. Methods Mol Biol. 2010;659:3-20.

[31] Arboleda VA, Lee H, Sánchez FJ, Délot EC, Sandberg DE, Grody WW, et al. Targeted massively parallel sequencing provides comprehensive genetic diagnosis for patients with disorders of sex development. Clin Genet. 2013 en.; 83(1):35-43.

[32] Hiort O, Birnbaum W, Marshall L, Wünsch L, Werner $\mathrm{R}$, Schröder T, et al. Management of disorders of sex development. Nat Rev Endocrinol. 2014 sept.;10(9):520-9.

[33] Barseghyan H, Délot E, Vilain E. New genomic technologies: an aid for diagnosis of disorders of sex development. Horm. Metab Res. 2015 may.;47(5):312-320.

[34] Baxter RM, Arboleda VA, Lee H, Barseghyan H, Adam MP, Fechner PY, et al. Exome sequencing for the diagnosis of 46,XY disorders of sex development. J Clin Endocrinol Metab. 2015 feb.;100(2):E333-44.

[35] Achermann JC, Domenice S, Bachega TA, Nishi MY, Mendonca BB. Disorders of sex development: effect of molecular diagnostics. Nat Rev Endocrinol. 2015 ag.;11(8):478-488. 